\title{
Exploiting A Priori Time Constant Ratio Information in Difference Equation Two-Thermocouple Sensor Characterization
}

\author{
Seán McLoone, Senior Member, IEEE, Peter Hung, Member, IEEE, George Irwin, Fellow, IEEE, and Robert J. Kee
}

\begin{abstract}
The characterization of thermocouple sensors for temperature measurement in varying-flow environments is a challenging problem. Recently, the authors introduced novel difference-equation-based algorithms that allow in situ characterization of temperature measurement probes consisting of two-thermocouple sensors with differing time constants. In particular, a linear least squares (LS) $\lambda$ formulation of the characterization problem, which yields unbiased estimates when identified using generalized total LS, was introduced. These algorithms assume that time constants do not change during operation and are, therefore, appropriate for temperature measurement in homogenous constant-velocity liquid or gas flows. This paper develops an alternative $\boldsymbol{\beta}$ formulation of the characterization problem that has the major advantage of allowing exploitation of a priori knowledge of the ratio of the sensor time constants, thereby facilitating the implementation of computationally efficient algorithms that are less sensitive to measurement noise. A number of variants of the $\beta$ formulation are developed, and appropriate unbiased estimators are identified. Monte Carlo simulation results are used to support the analysis.
\end{abstract}

Index Terms-Sensor characterization, soft sensing, twothermocouple probe (TTP).

\section{NOMENCLATURE}

$T_{g}(t) \quad$ True gas temperature (in degrees Celsius).

$T_{m}(t) \quad$ Measured gas temperature (in degrees Celsius).

$T_{1}(t) \quad$ Temperature measured by thermocouple 1 (in degrees Celsius).

$T_{2}(t) \quad$ Temperature measured by thermocouple 2 (in degrees Celsius).

$T_{1}^{k} \quad$ Temperature measured by thermocouple 1 at the $k$ th sample instant (in degrees Celsius).

$T_{2}^{k} \quad$ Temperature measured by thermocouple 2 at the $k$ th sample instant (in degrees Celsius).

$T_{g}^{k} \quad$ True gas temperature at the $k$ th sample instant (in degrees Celsius).

$T_{s} \quad$ Sampling period (in seconds).

Manuscript received November 28, 2005; revised April 19, 2006. The associate editor coordinating the review of this paper and approving it for publication was Prof. Ralph Etienne-Cummings.

S. McLoone and P. Hung are with the Department of Electronic Engineering, National University of Ireland Maynooth, Maynooth, Ireland (e-mail: s.mcloone@ieee.org; phung@eeng.nuim.ie).

G. Irwin is with the Virtual Engineering Centre, Queen's University Belfast, BT9 5HN Belfast, U.K. (e-mail: g.irwin@qub.ac.uk).

R. J. Kee is with the School of Mechanical and Aerospace Engineering, Queen's University Belfast, BT9 5AH Belfast, U.K. (e-mail: r.kee@qub.ac.uk).

Color versions of Figs. 2-4, 6, 8, and 9 are available online at http://ieeexplore.ieee.org.

Digital Object Identifier 10.1109/JSEN.2006.883906 $\tau_{1}$

$\tau_{2}$

$\alpha$

$v_{1}$

$v_{2}$

$\phi$

$a_{1}, b_{1}$

$a_{2}, b_{2}$

$\beta$

$\Delta T_{i}^{k}$

$\Delta T_{i j}^{k}$

$\boldsymbol{x}_{k}, y_{k}, \theta$

$\mathbf{X}, \boldsymbol{y}$

C

TTP

LS

RTLS

GTLS

SVD
Time constant of thermocouple 1 (in seconds).

Time constant of thermocouple 2 (in seconds).

Ratio of time constants, $\alpha=\tau_{1} / \tau_{2}, \alpha<1$ by definition.

Variance of noise on measurements from thermocouple 1.

Variance of noise on measurements from thermocouple 2 .

Ratio of the noise variances, $\phi=v_{1} / v_{2}$.

Parameters of the discrete-time model for thermocouple 1.

Parameters of the discrete-time model for thermocouple 2.

Ratio of $b_{2}$ and $b_{1}, \beta=b_{2} / b_{1}$.

Change in measured temperature from the $(k-1)$ th to the $k$ th sample instant for the $i$ th thermocouple, i.e., $\Delta T_{i}^{k}=T_{i}^{k}-T_{i}^{k-1}$ (in degrees Celsius).

Difference in temperature measured by the $i$ th and $j$ th thermocouple at the $k$ th sample instant, i.e., $\Delta T_{i j}^{k}=T_{i}^{k}-T_{j}^{k}$ (in degrees Celsius).

Generic regression, output vector, and parameter vector in $y_{k}=\boldsymbol{x}_{k}^{T} \theta$.

Matrix form of the equation $\boldsymbol{y}=\mathbf{X} \theta$.

Noise covariance matrix.

Two-thermocouple probe.

Least squares.

Restricted total LS.

Generalized total LS.

Singular value decomposition.

\section{INTRODUCTION}

F TWO dissimilar conducting wires are connected together to form a junction, a small voltage may be observed across the free ends, which is a function of the temperature of the junction. This thermoelectric effect, which was discovered by the Estonian physicist Thomas Johann Seebeck in 1821 (and which bears his name), is the fundamental operating principle of the most widely used temperature sensing device in commercial applications-the ubiquitous thermocouple. The popularity of the thermocouple stems from its simplicity, low cost, robustness, ease of manufacture, reliability, and wide operating range.

However, due to their relatively slow response times, thermocouples are only appropriate in applications where the 
temperature changes relatively slowly $(<1 \mathrm{~Hz})$. For higher frequency temperature fluctuations, fast response measurement systems, such as coherent anti-Stokes spectroscopy, laserinduced fluorescence, and infrared pyrometry, can be used, but such systems are expensive, are difficult to use and maintain in harsh environments, lack robustness, and are not practical for most commercial applications. An attractive alternative is to increase the effective bandwidth of thermocouples using software-based compensation techniques. Such techniques rely on having a dynamic model of the sensor whose inversion allows the true signal to be estimated from the measured output. For example, a linear first-order model with time constant $\tau$ is generally assumed in the case of temperature measurement in a gas or liquid flow [11], i.e.,

$$
\tau \frac{d T_{m}}{d t}+T_{m}(t)=T_{g}(t)
$$

where $T_{g}$ is the true gas temperature, and $T_{m}$ is the measured temperature.

Determining the time constant $\tau$, which is referred to as sensor characterization, is a critical step in the application of compensation schemes. This is normally achieved through an initial calibration procedure, where the thermocouple is heated by passing a current through it and then allowed to cool down in the environment in which it is being used. The time constant can then be estimated from the resulting cooling curve. The difficulty with this type of approach to calibration is that the time constant is strongly dependent on the physical and mechanical properties of the thermocouple and its environment, and therefore, a priori characterization is only applicable when these conditions do not change during sensor operation. In many situations, such as the measurement of temperature in a varying-flow environment, this is not the case. Here, the time constant of a thermocouple is related to its diameter according to

$$
\tau=k d^{2-m} v_{g}^{-m}
$$

where $k$ and $m$ are constants, approximately, arising from thermodynamic considerations, $d$ is the diameter of the thermocouple wire, and $v_{g}$ is the velocity of the gas/liquid medium in which it is placed.

In 1936, a German engineer, Hans Pfreim, discovered that by using a probe consisting of two thermocouples with different time constants, it is possible to identify both time constants in situ and subsequently reconstruct the input temperature [13]. The underlying assumption is that due to their close proximity, both thermocouples are subject to the same environmental conditions; hence, they have the same temperature $T_{g}(t)$ and medium velocity $v_{g}$. Under these circumstances, it follows from (2) that the ratio of the time constants, which is given by

$$
\alpha=\frac{\tau_{1}}{\tau_{2}}=\frac{k d_{1}^{2-m} v_{g}^{-m}}{k d_{2}^{2-m} v_{g}^{-m}}=\left(\frac{d_{1}}{d_{2}}\right)^{2-m}, \quad \alpha<1
$$

is a function of thermocouple geometry only and, therefore, approximately invariant. Here, the subscripts 1 and 2 are used to distinguish between the two thermocouples, and the corresponding thermocouple models are given by

$$
\begin{aligned}
\tau_{1} \frac{d T_{1}}{d t}+T_{1}(t) & =T_{g}(t) \\
\tau_{2} \frac{d T_{2}}{d t}+T_{2}(t) & =T_{g}(t)
\end{aligned}
$$

respectively. Assuming knowledge of $\alpha$, in situ instantaneous estimates of the time constants are given by

$$
\tau_{2}(t)=\frac{T_{1}(t)-T_{2}(t)}{\dot{T}_{2}(t)-\alpha \dot{T}_{1}(t)} \quad \tau_{1}(t)=\alpha \tau_{2}(t) .
$$

This novel idea was rediscovered by Strahle and Muthukrishman in 1976 and then again by Cambray in 1986. Strahle and Muthukrishman [14] developed a procedure for estimating the time constants in situ by analyzing the cross and auto power spectra of the probe signals, whereas Cambray [1] exploited the invariance of the time constant ratio $\alpha$ to reduce the problem to one of a priori ratio estimation. In particular, Cambray observed that $\alpha$ can be computed as the ratio of the slopes of the temperature responses $T_{1}(t)$ and $T_{2}(t)$ at crossover points, i.e.,

$$
\alpha=\frac{\dot{T}_{2}(t)}{\dot{T}_{1}(t)}, \quad \text { when } T_{1}(t)=T_{2}(t) .
$$

In recent years, these concepts have been developed further to produce more robust TTP in situ characterization and signal reconstruction algorithms. In [10]-[12], [16], and [17], timedomain methods have been developed, whereas in [2]-[4] and [15], the problem has been transformed to the frequency domain using the fast Fourier transform, thereby avoiding the numerical drawbacks associated with the estimation of derivatives. Tagawa et al. [15]-[17] have the added advantage of not requiring a priori time constant ratio information. All of the proposed methods give improved performance, but they have a number of weaknesses. Their performance deteriorates rapidly as the signal-to-noise ratio decreases. Some require accurate a priori estimation of the time constant ratio and are susceptible to numerical issues such as singularities. They are also sensitive to offsets and are not guaranteed to produce unbiased estimates in the presence of measurement noise.

Recently, the authors proposed a novel difference equation formulation of the TTP characterization problem that does not require the invariant $\alpha$ assumption and allows the problem to be cast as a linear input-output system identification problem whose parameters are algebraically related to the desired time constants, subject to a zero-order-hold $(\mathrm{ZOH})$ approximation [6]-[8]. In particular, a linear LS formulation of the characterization problem, which yields unbiased estimates when identified using GTLS, was introduced.

A weakness of the new difference equation approach is that the model parameters have no physical meaning, hence, a priori knowledge of $\alpha$ and its invariance cannot be exploited. In this paper, an alternative $\beta$ formulation of the characterization problem, which has the major advantage of allowing exploitation of a priori knowledge of $\alpha$, is described. A number of variants 


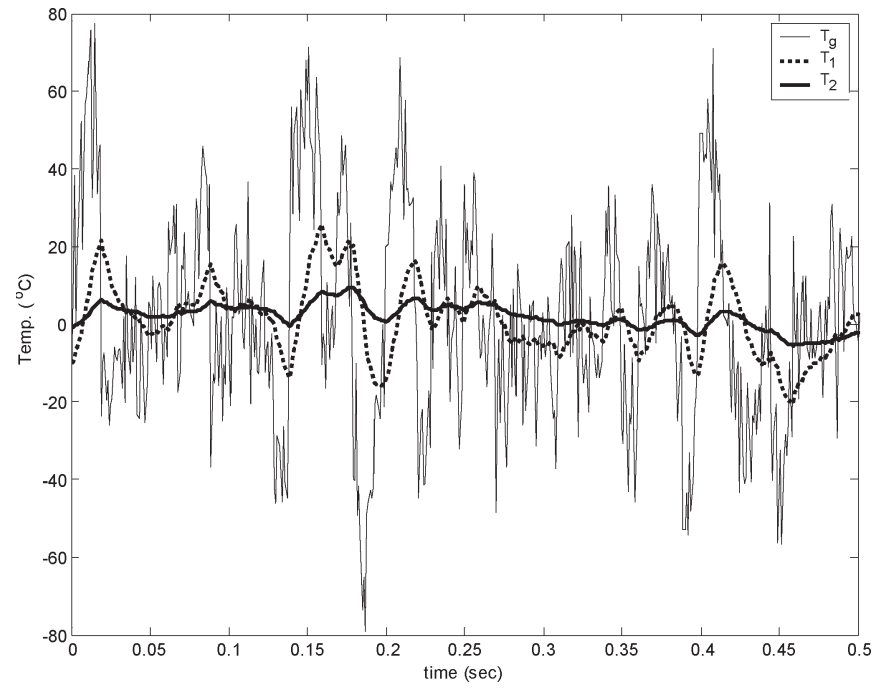

Fig. 1. Simulated gas temperature $T_{g}$ and thermocouple measurements $T_{1}$ and $T_{2}$.

of the formulation are developed, and appropriate unbiased estimators are identified. The issue of temperature offsets is also addressed. Monte Carlo simulation results are used to support the analysis.

A simulated TTP with 0.02 and $0.1 \mathrm{~s}$ time constant thermocouples measuring a broadband temperature signal is used throughout this paper as a benchmark problem to demonstrate the properties of the various algorithms developed. The input temperature fluctuations are modeled as band-limited white noise $(0-1 \mathrm{kHz})$ and sinusoidal tones at 15, 20, 25, 30, and $35 \mathrm{~Hz}$. A segment of the signal and corresponding thermocouple measurements is illustrated in Fig. 1. The thermocouple signals are sampled at $1 \mathrm{kHz}$ and zero-mean normally distributed random numbers added to the samples to simulate white measurement noise. The noise level, which is defined as

$$
\text { Noise level }=\sqrt{\frac{\text { Noise power }}{\text { Signal power }}} \times 100
$$

is used to quantify the amount of noise introduced.

The remainder of this paper is structured as follows: Section II introduces some preliminaries on LS optimization focusing on the problem of bias-free estimation. The TTP discrete-time characterization approach and linear LS $\lambda$ formulation are then outlined in Section III. Section IV develops the novel $\beta$ formulation and an extension to address temperature offsets. Monte Carlo simulation results are given in Section V, and finally, conclusions are presented in Section VI.

\section{LS PRELIMINARIES}

\section{A. $L S$}

In the conventional LS formulation, we have a linear model of the form

$$
y_{k}=\boldsymbol{x}_{k}^{T} \theta
$$

where $\boldsymbol{x}_{k}$ is the $p \times 1$ regression vector, $y_{k}$ is a scalar output, and $\theta$ is the $p \times 1$ vector of unknown parameters. For a set of $n$ samples, the regression matrix and corresponding output vector can be defined as

$$
\mathbf{X}=\left[\boldsymbol{x}_{1} \cdots \boldsymbol{x}_{n}\right]^{T} \quad \boldsymbol{y}=\left[y_{1} \cdots y_{n}\right]^{T}
$$

leading to the matrix equation

$$
\boldsymbol{y}=\mathbf{X} \theta
$$

Here, $\mathbf{X}$ is an $n \times p$ matrix, and $\boldsymbol{y}$ is an $n \times 1$ vector. The LS estimate of $\theta$ is then given by

$$
\theta_{\mathrm{LS}}=\left[\mathbf{X}^{T} \mathbf{X}\right]^{-1} \mathbf{X}^{T} \boldsymbol{y}=\mathbf{X}^{\dagger} \boldsymbol{y}
$$

Due to numerical issues, the pseudoinverse $\left(\mathbf{X}^{\dagger}\right)$ is seldomly computed directly. Instead, robust procedures such as SVD are employed [5].

\section{B. Statistical Properties of the LS Estimate}

To evaluate the statistical properties of the LS solution in the presence of noise, it is useful to express the solution in terms of the sample covariance matrix $\mathbf{R}_{n}$ and the sample cross correlation vector $\boldsymbol{p}_{n}$ as

$$
\mathbf{R}_{n}=\frac{\mathbf{X}^{T} \mathbf{X}}{n} \quad \boldsymbol{p}_{n}=\frac{\mathbf{X}^{T} \boldsymbol{y}}{n} .
$$

By definition, as the number of data points $n$ tends to infinity, we obtain the true correlation matrix and cross correlation vector, i.e.,

$$
\mathbf{R}=\lim _{n \rightarrow \infty} \mathbf{R}_{n}=E\left(\boldsymbol{x}_{k} \boldsymbol{x}_{k}^{T}\right) \quad \boldsymbol{p}=\lim _{n \rightarrow \infty} \boldsymbol{p}_{n}=E\left(y_{k} \boldsymbol{x}_{k}^{T}\right) .
$$

Using these definitions, the least square estimate (12) can now be expressed as

$$
\theta_{\mathrm{LS}}=\mathbf{R}_{n}^{-1} \boldsymbol{p}_{n}
$$

If the regressor (i.e., $\boldsymbol{x}_{k}=\left[x_{k}^{1} \cdots x_{k}^{p}\right]^{T}$ ) and output (i.e., $y_{k}$ ) measurements are subject to zero-mean random noise, i.e.,

$$
\widetilde{x}_{k}^{i}=x_{k}^{i}+n_{k}^{i} \quad \widetilde{y}_{k}=y_{k}+w_{k} \quad \boldsymbol{n}_{k}=\left[n_{k}^{1} \ldots n_{k}^{p}\right]^{T}
$$

where

$$
E\left[n_{k}^{i}\right]=0 \quad E\left[\left(n_{k}^{i}\right)^{2}\right]=v_{i} \quad E\left[w_{k}\right]=0 \quad E\left[w_{k}^{2}\right]=v_{y}
$$

then analysis of the LS estimate shows that its expected value is given by

$$
E\left[\widetilde{\theta}_{\mathrm{LS}}\right]=\left[\mathbf{R}+\mathbf{C}_{\mathbf{X X}}\right]^{-1}\left(\boldsymbol{p}+\boldsymbol{c}_{\mathbf{X} y}\right)
$$

where $\mathbf{C}_{\mathbf{X X}}=E\left[\boldsymbol{n}_{k} \boldsymbol{n}_{k}^{T}\right]$ and $\boldsymbol{c}_{\mathbf{X} y}=E\left[\boldsymbol{n}_{k} w_{k}\right]$. Thus, in this general case, the LS estimate is strongly biased. In fact, the only situation where LS produces unbiased estimates is when zeromean noise is present on the output only, and the regressors are 
noise free. However, an alternative approach known as GTLS can be used to get an unbiased estimate of $\theta$ when noise is present on both the regressors and output, provided the overall noise covariance matrix

$$
\mathbf{C}=E\left[\left[\begin{array}{c}
\boldsymbol{n}_{k} \\
w_{k}
\end{array}\right]\left[\begin{array}{ll}
\boldsymbol{n}_{k}^{T} & w_{k}
\end{array}\right]\right]=\left[\begin{array}{cc}
\mathbf{C}_{\mathbf{X X}} & \boldsymbol{c}_{\mathbf{X} y} \\
\boldsymbol{c}_{\mathbf{X} y}^{T} & v_{y}
\end{array}\right]
$$

is known up to a factor of proportionality, i.e., $\mathbf{C}=v \mathbf{C}_{0}$. Given $\mathbf{C}_{0}$ and the noisy regression matrix and output vector data $\widetilde{\mathbf{X}}$ and $\widetilde{\boldsymbol{y}}$, the GTLS solution $\theta_{\mathrm{GTLS}}$ can be computed in a robust fashion from the generalized SVD [18] of the matrix pair $[\widetilde{\mathbf{X}} \widetilde{\boldsymbol{y}}]$ and $\sqrt{\mathbf{C}_{0}}$, i.e.,

$$
\operatorname{gsvd}\left([\widetilde{\mathbf{X}} \widetilde{\boldsymbol{y}}], \sqrt{\mathbf{C}_{0}}\right) \rightarrow\left\{\begin{array}{l}
{[\widetilde{\mathbf{X}} \widetilde{\boldsymbol{y}}]=\mathbf{U} \boldsymbol{\Sigma}_{\mathbf{X} y} \mathbf{G}^{-1}} \\
\sqrt{\mathbf{C}_{0}}=\mathbf{V} \boldsymbol{\Sigma}_{\mathbf{C}} \mathbf{G}^{-1} \\
\boldsymbol{\Sigma}^{2}=\boldsymbol{\Sigma}_{\mathbf{X} y}^{T} \boldsymbol{\Sigma}_{\mathbf{X} y}\left[\boldsymbol{\Sigma}_{\mathbf{C}}^{T} \boldsymbol{\Sigma}_{\mathbf{C}}\right]^{-1}
\end{array} .\right.
$$

Here, $\sqrt{\mathbf{C}_{0}}$ denotes the Cholesky decomposition of $\mathbf{C}_{0}$, $\boldsymbol{\Sigma}_{\mathbf{C}} \in \Re^{(p+1) \times(p+1)}$, and $\boldsymbol{\Sigma}_{\mathbf{X} y} \in \Re^{(p-1) \times(p+1)}$ are diagonal matrices with singular values on their diagonals, $\boldsymbol{\Sigma}$ contains the generalized singular values and matrix $\mathbf{G}=\left[\boldsymbol{g}_{1} \cdots \boldsymbol{g}_{p+1}\right]$ contains the corresponding generalized singular vectors. The GTLS solution is then given by

$$
\theta_{\mathrm{GTLS}}=-\frac{\boldsymbol{g}_{p+1}}{g_{p+1, p+1}}
$$

where $\boldsymbol{g}_{p+1}$ is the generalized singular vector associated with the smallest generalized singular value.

\section{Difference Equation TTP Technique}

\section{A. Basic Principles}

For a given sampling interval $T_{s}$, the thermocouples constituting a TTP can be modeled as first-order difference equations of the form

$$
\begin{aligned}
& T_{1}^{k}=a_{1} T_{1}^{k-1}+b_{1} T_{g}^{k-1} \\
& T_{2}^{k}=a_{2} T_{2}^{k-1}+b_{2} T_{g}^{k-1} .
\end{aligned}
$$

These can be related to the continuous-time equations describing the TTP [i.e., (4) and (5)] under the assumption of $\mathrm{ZOH}$ on the input signal $T_{g}$, i.e.,

$$
a_{i}=\exp \left(-\frac{T_{s}}{\tau_{i}}\right) \quad b_{i}=1-a_{i}, \quad i=1,2 .
$$

While $\mathrm{ZOH}$ is clearly not true for a continuously changing gas temperature, it becomes a valid approximation, provided the system is sufficiently oversampled. Thus, if the parameters of the discrete model equations can be determined, the thermocouple time constants can be estimated as

$$
\tau_{i}=\frac{T_{s}}{\ln \left(a_{i}\right)}, \quad i=1,2 .
$$

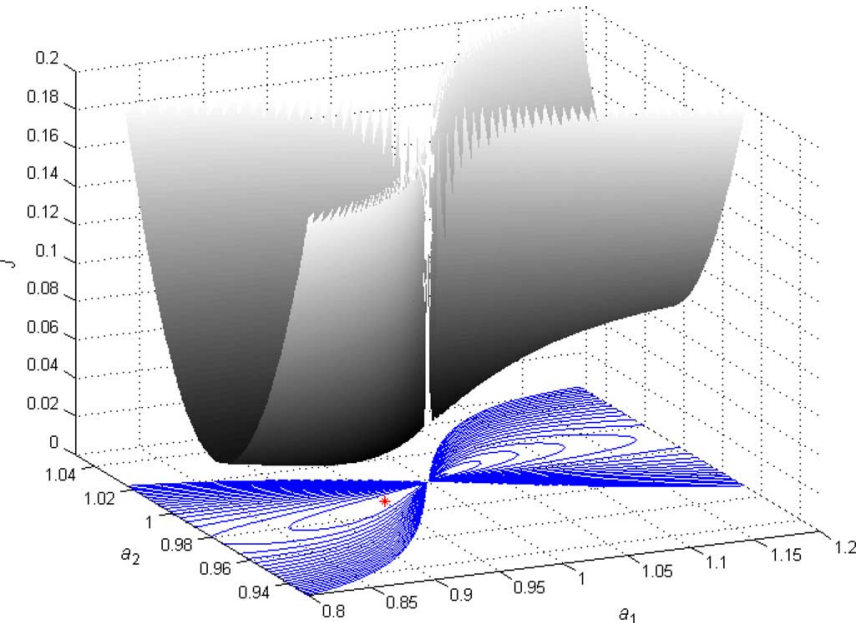

Fig. 2. TTP characterization cost function $J_{a}\left(a_{1}, a_{2}\right)$.

Unknown signal $T_{g}^{k-1}$ can be eliminated from the simultaneous equations formed by (22) and (23) to give the difference equation TTP model as

$$
T_{2}^{k}=a_{2} T_{2}^{k-1}+\left(\frac{1-a_{2}}{1-a_{1}}\right) T_{1}^{k}-a_{1}\left(\frac{1-a_{2}}{1-a_{1}}\right) T_{1}^{k-1} .
$$

This is a nonlinear in the parameter model and must be solved using nonlinear methods. This typically involves minimizing an MSE cost function of the form

$$
\begin{array}{r}
J_{a}\left(a_{1}, a_{2}\right)=\frac{1}{n} \sum_{i=2}^{n+1}\left[\left(T_{2}^{i}-a_{2} T_{2}^{i-1}-\left(\frac{1-a_{2}}{1-a_{1}}\right) T_{1}^{i}\right.\right. \\
\left.\left.+a_{1}\left(\frac{1-a_{2}}{1-a_{1}}\right) T_{1}^{i-1}\right)^{2}\right] .
\end{array}
$$

A plot of this cost function for the simulated TTP benchmark is given in Fig. 2. Note that the cost function is highly nonlinear with a singularity at $a_{1}=1$. While there is only a single global minimum, minimization by iterative gradient-based methods is poorly conditioned, leading to slow convergence and numerical issues. In addition, minimization in the presence of noise is biased, but there is no systematic approach for dealing with this in a nonlinear setting. Consequently, alternative linear formulations are needed.

\section{B. $\lambda$ Formulation}

In [7], the authors proposed a linear three-parameter $\lambda$ formulation of the problem, where (26) is written as

$$
T_{2}^{k}=\lambda_{1} T_{2}^{k-1}+\lambda_{2} T_{1}^{k}+\lambda_{3} T_{1}^{k-1} .
$$

This corresponds to identifying the discrete-time model given in Fig. 3. Following identification of the linear model parameters $\lambda_{1}, \lambda_{2}$, and $\lambda_{3}$, the desired coefficients $a_{1}$ and $a_{2}$ can be determined according to

$$
a_{1}=-\frac{\lambda_{3}}{\lambda_{2}} \quad a_{2}=\lambda_{1} .
$$




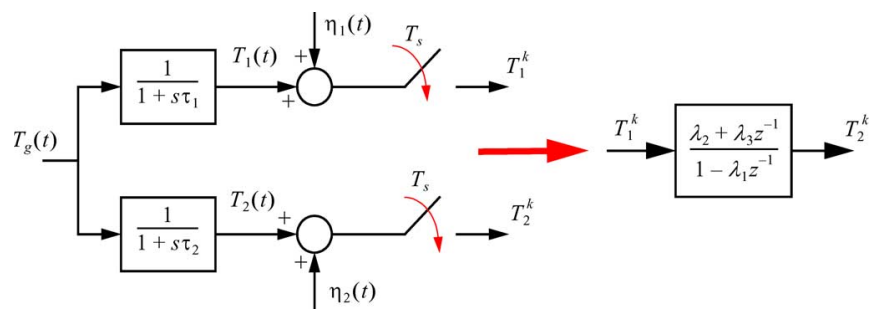

Fig. 3. Discrete-time TTP formulation.

If the measured temperatures $T_{1}$ and $T_{2}$ are subject to zeromean identically distributed white noise with variances $v_{1}$ and $v_{2}$, respectively, then unbiased estimates of the parameters can be obtained using GTLS, provided the ratio of the noise variances, i.e., $\phi=v_{1} / v_{2}$ is known. Under these conditions, the required noise covariance matrix, as defined in (19), is given by

$$
\mathbf{C}_{\lambda_{3}}=\operatorname{diag}\left(v_{2}, v_{1}, v_{1}, v_{2}\right)=v_{2} \operatorname{diag}(1, \phi, \phi, 1) .
$$

This approach introduces an extra degree of freedom into the estimation process, leading to increased estimation variance and the possibility of inconsistent time constant estimates at high noise levels.

However, noting that the constraint on the extra degree of freedom can be expressed as

$$
\lambda_{1}+\lambda_{2}+\lambda_{3}=1
$$

substitution of the constraint into the identification model in (28) reduces parameter estimation to a two-dimensional linear optimization problem, the exact form of which depends on which of the three unknown parameters is eliminated. For example, if $\lambda_{3}$ is eliminated by substituting

$$
\lambda_{3}=1-\lambda_{1}-\lambda_{2}
$$

then (28) becomes

$$
\begin{gathered}
T_{2}^{k-T_{1}^{k-1}}=\lambda_{1}\left(T_{2}^{k-1}-T_{1}^{k-1}\right)+\lambda_{2}\left(T_{1}^{k}-T_{1}^{k-1}\right) \\
{\left[T_{2}^{k-T_{1}^{k-1}}\right]=\left[\begin{array}{ll}
T_{2}^{k-1}-T_{1}^{k-1} & T_{1}^{k}-T_{1}^{k-1}
\end{array}\right]\left[\begin{array}{c}
\lambda_{1} \\
\lambda_{2}
\end{array}\right] .}
\end{gathered}
$$

The form of the bias in the LS solution is more complex in this formulation due to the multiple occurrences of $T_{1}$ and $T_{2}$ in the regressor and output. If we assume zero-mean noise with variances $v_{1}$ and $v_{2}$ as before, then the resulting estimates of the correlation matrix and cross correlation vector are

$$
E\left[\widetilde{\mathbf{R}}_{n}\right]=\mathbf{R}+\left[\begin{array}{cc}
v_{1}+v_{2} & v_{1} \\
v_{1} & 2 v_{1}
\end{array}\right] \quad E\left[\widetilde{\boldsymbol{p}}_{n}\right]=\boldsymbol{p}+\left[\begin{array}{l}
v_{1} \\
v_{1}
\end{array}\right]
$$

and the overall noise covariance matrix is given by

$$
\mathbf{C}_{\lambda_{2}}=v_{2}\left[\begin{array}{ccc}
\phi+1 & \phi & \phi \\
\phi & 2 \phi & \phi \\
\phi & \phi & \phi+1
\end{array}\right] .
$$

Thus, provided the noise variance ratio $\phi$ is known or can be estimated, GTLS can be used to obtain unbiased parameter estimates.
Linear two-parameter equations can also be obtained by eliminating $\lambda_{1}$ or $\lambda_{2}$, but since the different formulations are related algebraically in a manner that is independent of the measurements, it follows that the statistical properties are not affected by these transformations. Hence, the bias and covariance of the time constant estimates obtained is the same for all the two-parameter formulations.

\section{IV. $\beta$ FORMULATIONS}

Now, consider the scenario where the ratio of the time constants $\alpha$ is known. This cannot be exploited in the discrete-time formulations considered thus far, as they are not parameterized in terms of time constants. However, an equivalent discrete-time parameter ratio $\beta$ can be derived as follows.

If $T_{s} / \tau_{i} \ll 1$, a situation that is desirable for the validity of the $\mathrm{ZOH}$ approximation underpinning the difference equation characterization methods, (24) can be approximated as

$$
a_{i}=\exp \left(-\frac{T_{s}}{\tau_{i}}\right) \approx 1-\frac{T_{s}}{\tau_{i}}
$$

and since $b_{i}=1-a_{i}$, it follows that

$$
b_{i} \approx \frac{T_{s}}{\tau_{i}} .
$$

Hence, the discrete-time parameter ratio $\beta$, which is defined as $b_{2} / b_{1}$, is approximately equal to the time constant ratio, i.e.,

$$
\beta=\frac{b_{2}}{b_{1}} \approx \frac{T_{s} / \tau_{2}}{T_{s} / \tau_{1}}=\frac{\tau_{1}}{\tau_{2}}=\alpha .
$$

It is important to note that while $\alpha$ may be constant, $\beta$ does vary as a function of time constants, i.e.,

$$
\beta\left(\frac{T_{s}}{\tau_{1}}\right)=\frac{1-\exp \left(-\alpha T_{s} / \tau_{1}\right)}{1-\exp \left(-T_{s} / \tau_{1}\right)} .
$$

This is illustrated in Fig. 4(a), which shows a plot of $\beta$ as a function of $\tau_{1}$ for different sampling intervals when $\alpha=0.2$. The corresponding sensitivity function, which is defined as the ratio of the relative change in $\beta$ to the relative change in $\tau_{1}$ and is given by

$$
\begin{aligned}
& S_{\tau_{1}}^{\beta}\left(\frac{T_{s}}{\tau_{1}}\right)=\frac{T_{s}}{\tau_{1}} \cdot\left[\frac{a_{1}}{b_{1}}-\alpha \frac{a_{2}}{b_{2}}\right] \\
&=\frac{T_{s}}{\tau_{1}} \cdot\left[\frac{\exp \left(-T_{s} / \tau_{1}\right)}{1-\exp \left(-T_{s} / \tau_{1}\right)}\right. \\
&\left.\quad-\alpha \frac{\exp \left(-\alpha T_{s} / \tau_{1}\right)}{1-\exp \left(-\alpha T_{s} / \tau_{1}\right)}\right]
\end{aligned}
$$

is also displayed in Fig. 4(b). An examination of these graphs shows that the error in the $\beta$ approximation to $\alpha$ is less than $0.5 \%$, provided $T_{s} / \tau_{1}<0.1$ and that the variation in $\beta$ is negligible when $T_{s} / \tau_{1}<0.01$. Note that for a given $\alpha$, the sensitivity of $\beta$ to variations in $\tau_{1}$ depends only on the ratio $T_{s} / \tau_{1}$; hence, $S_{\tau_{1}}^{\beta}$ is plotted as a function of $T_{s} / \tau_{1}$ rather than $\tau_{1}$ in Fig. 4(b). 


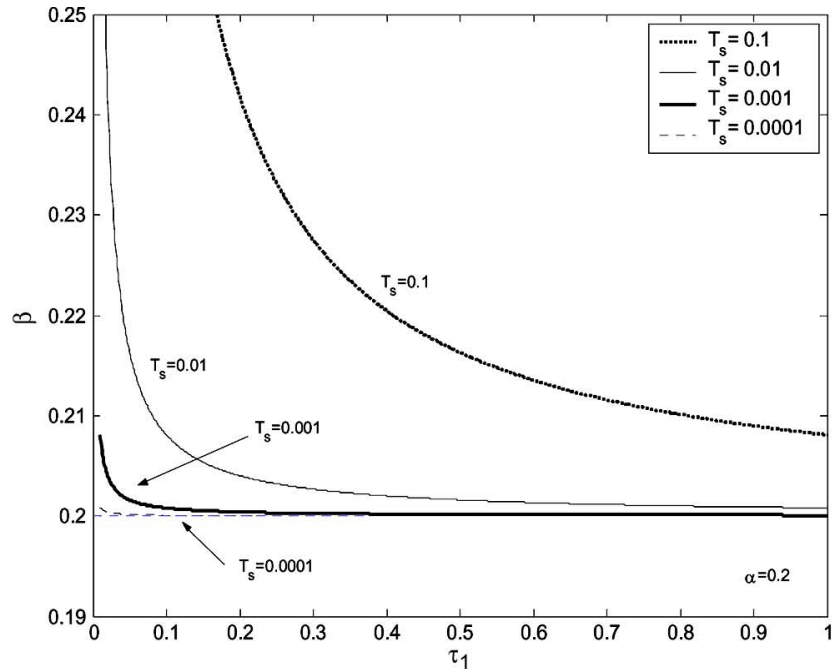

(a)

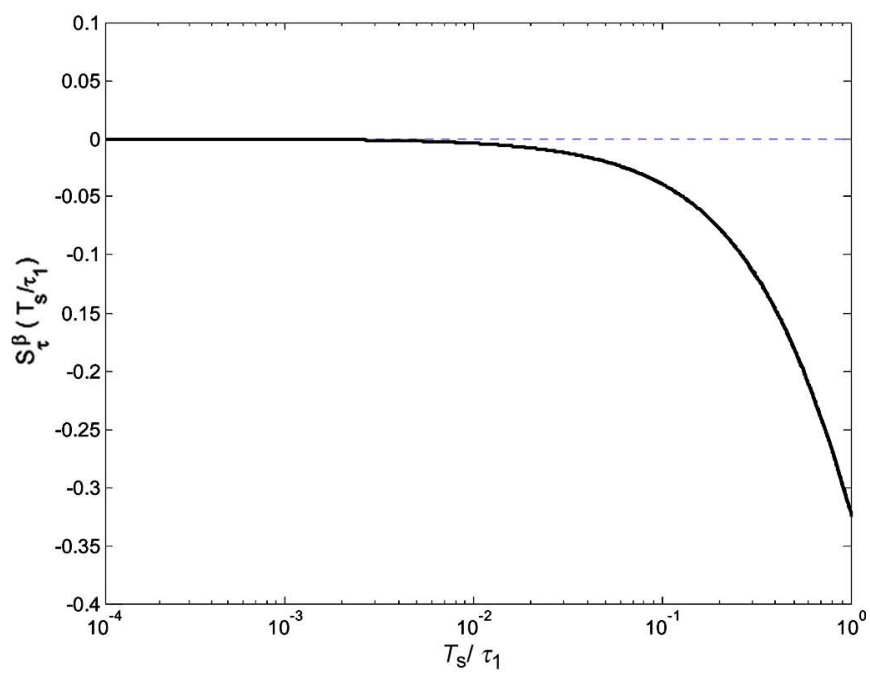

(b)

Fig. 4. Plots showing the insensitivity of $\beta$ to time-varying time constants. (a) $\beta$ as a function of $\tau_{1}$ with $\alpha=0.2$ for different sampling intervals $T_{s}$. (b) Sensitivity of $\beta$ to changes in $\tau_{1}$ as a function of $T_{s} / \tau_{1}(\alpha=0.2)$.

\section{A. Two-Parameter $\beta$ Algorithm}

Given this useful link between $\beta$ and $\alpha$, it is prudent to express the TTP characterization problem in terms of discrete parameters $b_{1}$ and $b_{2}$ instead of $a_{1}$ and $a_{2}$. Thus, substituting for $a_{1}$ and $a_{2}$ in (26) yields

$$
T_{2}^{k}=\left(1-b_{2}\right) T_{2}^{k-1}+\frac{b_{2}}{b_{1}} T_{1}^{k}-\left(1-b_{1}\right) \frac{b_{2}}{b_{1}} T_{1}^{k-1} .
$$

Again, this is nonlinear in terms of $b_{1}$ and $b_{2}$ and can be solved by minimizing a cost function of the form

$$
\begin{aligned}
J_{b}\left(b_{1}, b_{2}\right)=\frac{1}{n} \sum_{i=2}^{n+1}[ & \left(T_{2}^{i}-\left(1-b_{2}\right) T_{2}^{i-1}\right. \\
& \left.\left.\quad-\frac{b_{2}}{b_{1}} T_{1}^{i}+\left(1-b_{1}\right) \frac{b_{2}}{b_{1}} T_{1}^{i-1}\right)^{2}\right]
\end{aligned}
$$

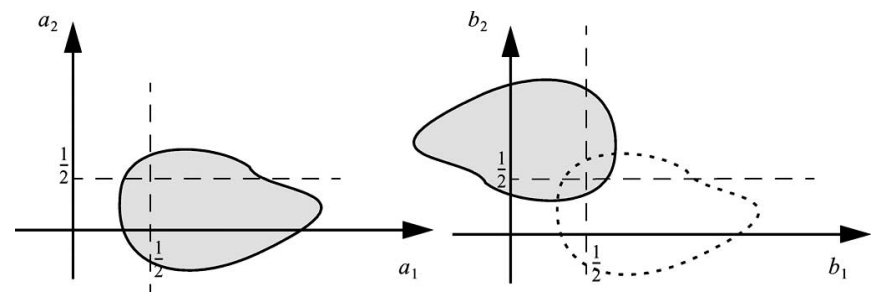

Fig. 5. Graphical illustration of relationship between $J_{a}$ and $J_{b}$.

using nonlinear optimization methods. However, there is no benefit to be gained from this reformulation since the resulting cost function is simply a reflection of $J_{a}\left(a_{1}, a_{2}\right)$ about the point $(1 / 2,1 / 2)$, i.e.,

$$
J_{b}(x, y)=J_{a}(1-x, 1-y) .
$$

This is illustrated graphically in Fig. 5.

However, introducing the ratio $\beta$ into (42) and noting that $b_{1} \beta=b_{2}$ allows the problem to be cast as a two-parameter linear equation, i.e.,

$$
T_{2}^{k}=\left(1-b_{2}\right) T_{2}^{k-1}+\beta T_{1}^{k}-\beta T_{1}^{k-1}+b_{2} T_{1}^{k-1} .
$$

Collecting terms and expressing in matrix-vector form gives

$$
\Delta T_{2}^{k}=\left[\begin{array}{ll}
\Delta T_{1}^{k} & \Delta T_{12}^{k-1}
\end{array}\right]\left[\begin{array}{c}
\beta \\
b_{2}
\end{array}\right] .
$$

Here, the notation $\Delta T_{i}^{k}=T_{i}^{k}-T_{i}^{k-1}$ and $\Delta T_{i j}^{k}=T_{i}^{k}-T_{j}^{k}$ is used for conciseness. Again, conventional LS estimation is biased with

$$
E\left[\widetilde{\mathbf{R}}_{n}\right]=\mathbf{R}+\left[\begin{array}{cc}
2 v_{1} & -v_{1} \\
-v_{1} & v_{1}+v_{2}
\end{array}\right] \quad E\left[\widetilde{\boldsymbol{p}}_{n}\right]=\boldsymbol{p}+\left[\begin{array}{c}
0 \\
v_{2}
\end{array}\right]
$$

but GTLS with

$$
\mathbf{C}_{\beta b_{2}}=v_{2}\left[\begin{array}{ccc}
2 \phi & -\phi & 0 \\
-\phi & \phi+1 & 1 \\
0 & 1 & 2
\end{array}\right]
$$

will give unbiased estimates. Note that the LS solution corresponds to minimizing

$$
J_{b_{2} \beta}\left(b_{2}, \beta\right)=\frac{1}{n} \sum_{i=2}^{n+1}\left[\left(\Delta T_{2}^{i}-\beta \Delta T_{1}^{i}-b_{2} \Delta T_{12}^{i-1}\right)^{2}\right]
$$

which is a nonlinear transformation of the cost function $J_{a}\left(a_{1}, a_{2}\right)$, i.e.,

$$
J_{b_{2} \beta}\left(b_{2}, \beta\right)=J_{a}\left(1-\frac{b_{2}}{\beta}, 1-b_{2}\right) .
$$

The significance of this transformation is that a nonquadratic cost function has been converted to a quadratic one (see Fig. 6), allowing the application of powerful linear optimization methods as well as a framework for unbiased estimation.

This two-parameter $\beta$ formulation is algebraically related to the $\lambda_{2}$ formulations described in Section III-B and, therefore, has equivalent numerical and statistical properties. Thus, all the two-parameter algorithms are of equal merit when no 


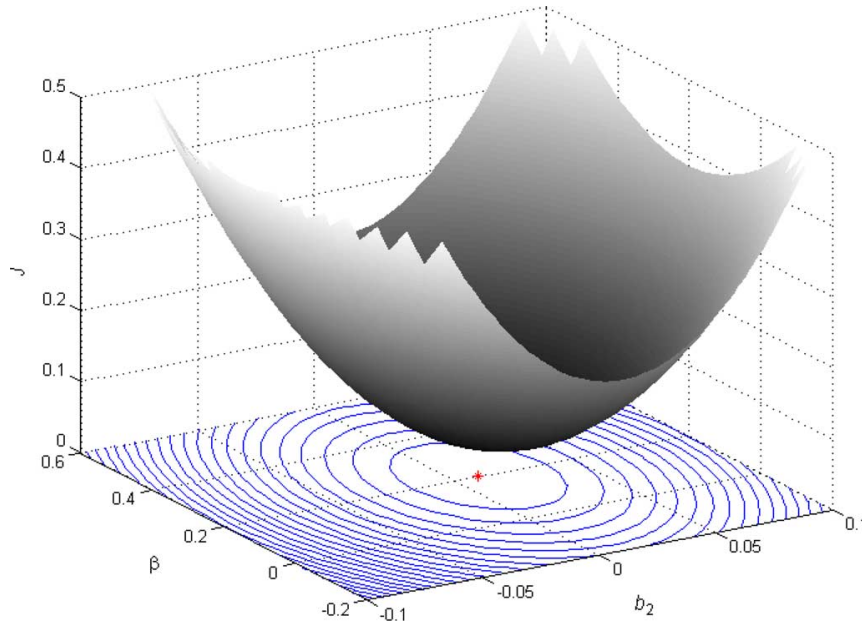

Fig. 6. Plot of $J_{b_{2} \beta}\left(b_{2}, \beta\right)$ for the simulated benchmark problem.

assumptions are made about the time constant ratio. However, when operating in a time-varying environment with real-time constraints, there may be scope for exploiting the invariance of $\beta$ to provide more stable and efficient recursive estimation algorithms. This is explored in [9], where a robust sliding window algorithm for estimating time-varying time constants is developed on the assumption that $\beta$ is constant over the data window and that the variation in $b_{2}$ can be approximated by a polynomial expansion.

\section{B. One-Parameter $\beta$ Algorithm}

The major benefit of the $\beta$ formulation arises when the time constant ratio $\alpha$ and, consequently, $\beta$, is known a priori. This allows TTP characterization to be reduced to a single parameter estimation problem, leading to a significant reduction in computational complexity. Collecting the known terms on the left-hand side, (46) can be rewritten as

$$
\Delta T_{2}^{k}-\beta \Delta T_{1}^{k}=b_{2} \Delta T_{12}^{k-1} .
$$

This is now a univariate linear optimization problem of the form $y_{k}=x_{k} b_{2}$, where

$$
x_{k}=\Delta T_{12}^{k-1} \quad y_{k}=\Delta T_{2}^{k}-\beta \Delta T_{1}^{k} .
$$

Given $n+1$ successive samples of $T_{1}$ and $T_{2}$, a computationally efficient LS estimate of $b_{2}$ can be derived as

$$
\widetilde{b}_{2}=\frac{\widetilde{\boldsymbol{z}}^{T} \widetilde{\boldsymbol{y}}}{\widetilde{\boldsymbol{z}}^{T} \widetilde{\boldsymbol{z}}}
$$

where $\widetilde{\boldsymbol{z}}=\left[\widetilde{x}_{1} \cdots \widetilde{x}_{n}\right]^{T}$ and $\widetilde{\boldsymbol{y}}=\left[\widetilde{y}_{1} \cdots \widetilde{y}_{n}\right]^{T}$ are the noisecorrupted regression and output data, respectively. Following a similar analysis to that outlined in Section II-B, it can be shown that the expected value of $\widetilde{b}_{2}$ is given by

$$
E\left[\widetilde{b}_{2}\right]=\frac{p+\beta v_{1}+v_{2}}{R+v_{1}+v_{2}}
$$

where $R$ and $p$ are the scalar equivalent to the covariance matrix and cross correlation vector. Since the true parameter value $b_{2}$ is given by $p / R$, it follows that LS estimation is strongly biased even in this univariate case. A compensated estimate can be computed if the variance of the measurement noise is known, i.e.,

$$
b_{2}^{*}=\frac{\widetilde{\boldsymbol{z}}^{T} \widetilde{\boldsymbol{y}}-n\left(\beta v_{1}+v_{2}\right)}{\widetilde{\boldsymbol{z}}^{T} \widetilde{\boldsymbol{z}}-n\left(v_{1}+v_{2}\right)} .
$$

Alternatively, at the expense of increased computational complexity, GTLS may also be used to obtain an unbiased estimate of $b_{2}$. The required noise covariance matrix has the form

$$
\mathbf{C}_{b_{2}}=\left[\begin{array}{cc}
v_{1}+v_{2} & \beta v_{1}+v_{2} \\
\beta v_{1}+v_{2} & 2 \beta^{2} v_{1}+2 v_{2}
\end{array}\right]=v_{2}\left[\begin{array}{cc}
\phi+1 & \beta \phi+1 \\
\beta \phi+1 & 2 \beta^{2} \phi+2
\end{array}\right] .
$$

An added advantage of employing the one-parameter formulation is that with only one degree of freedom, the estimation variance due to noise will be significantly reduced when compared to the two-parameter and three-parameter formulations. This is illustrated in Fig. 7, which shows the results of a 100-run Monte Carlo simulation analysis of GTLS estimates obtained using the one-parameter fixed- $\beta$, two-parameter $\beta-b_{2}$, and three-parameter $\lambda$ formulations for the simulated benchmark problem when the noise levels on thermocouples 1 and 2 are set at $2 \%$ and $4.2 \%$, respectively (i.e., $\phi=2$ ). Plots are given for the $\beta-b_{2}$ parameters and the corresponding time constants $\tau_{1}-\tau_{2}$. Covariance ellipses are included to highlight the distribution of the estimates.

1) Sensitivity to Errors in $\beta$ : An evaluation of the sensitivity of the $b_{2}$ and time constant estimates to errors in $\beta$ is presented in Fig. 8. The results are for the simulated example described earlier with the noise level on the measurements from thermocouples 1 and 2 set at 5\% and 10.5\%, respectively (i.e., $\phi=2$ ). The plots show the mean estimates and $95 \%$ confidence intervals computed on the basis of a 100-run Monte Carlo simulation. A number of significant patterns are evident. Parameter $b_{2}$ is relatively insensitive to errors in $\beta$ with an error of less than $2.5 \%$ introduced on average for a $50 \%$ error in $\beta$, i.e., $S_{b_{2}}^{\beta}<1 / 20$. A similar trend is observed with the larger of the time constants $\tau_{2}$. However, in sharp contrast to this, time constant $\tau_{1}$ is very sensitive to errors in $\beta$ with $S_{\tau_{1}}^{\beta} \approx 1$. Noting the approximate relationship between time constants and $b$ parameters (38), an analysis of the error propagation shows that

$$
S_{\tau_{1}}^{b_{1}}=S_{\tau_{2}}^{b_{2}}=1
$$

and since $b_{1}$ is computed as $b_{2} / \beta$, it follows that

$$
S_{b_{1}}^{\beta}=S_{b_{2}}^{\beta}+S_{\beta}^{\beta}=S_{b_{2}}^{\beta}+1 .
$$

Therefore, the time constant sensitivities to errors in $\beta$ can be expressed as

$$
S_{\tau_{1}}^{\beta}=S_{\tau_{1}}^{b_{1}} \cdot S_{b_{1}}^{\beta}=S_{b_{2}}^{\beta}+1 \quad S_{\tau_{2}}^{\beta}=S_{\tau_{2}}^{b_{2}} \cdot S_{b_{2}}^{\beta}=S_{b_{2}}^{\beta}
$$

indicating that $\tau_{2}$ errors are due to deviations in $b_{2}$, whereas $\beta$ inaccuracies are the dominant contribution to errors in $\tau_{1}$. 


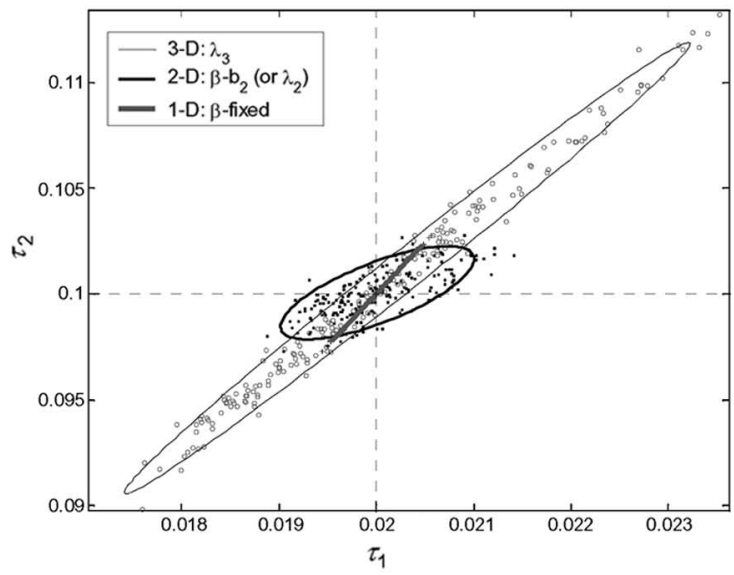

(a)

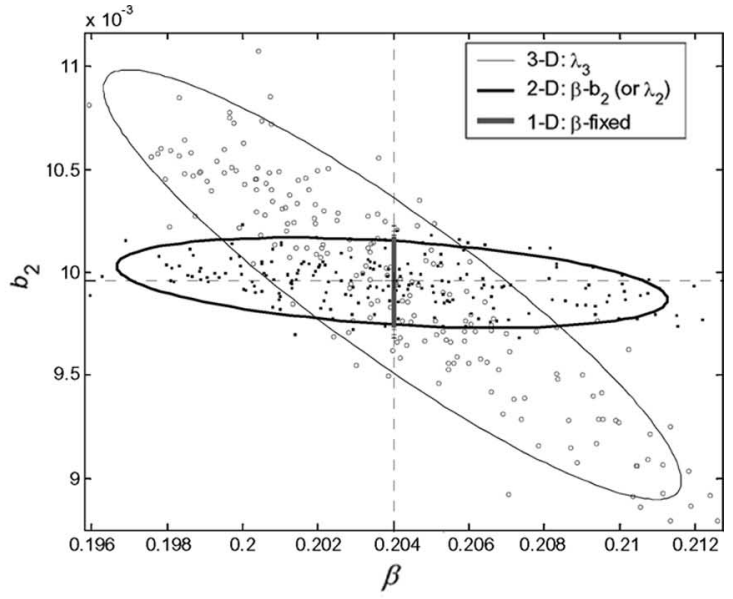

(b)

Fig. 7. Monte Carlo simulation GTLS parameter estimates and covariance ellipses for the one-, two-, and three-parameter TTP characterization formulations ( $2 \%$ and $4.2 \%$ thermocouple noise levels; $\phi=2$ ). (a) Monte Carlo time constant estimates. (b) Monte Carlo $\beta$ and $b_{2}$ estimates.

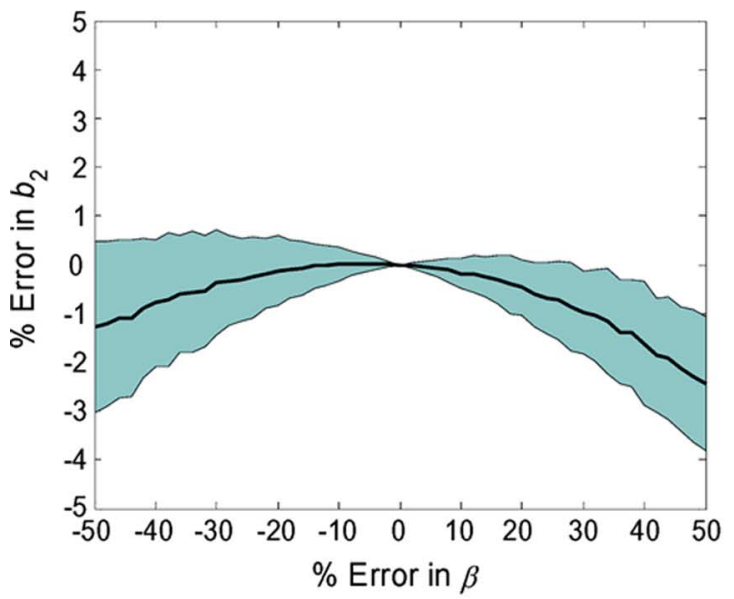

(a)

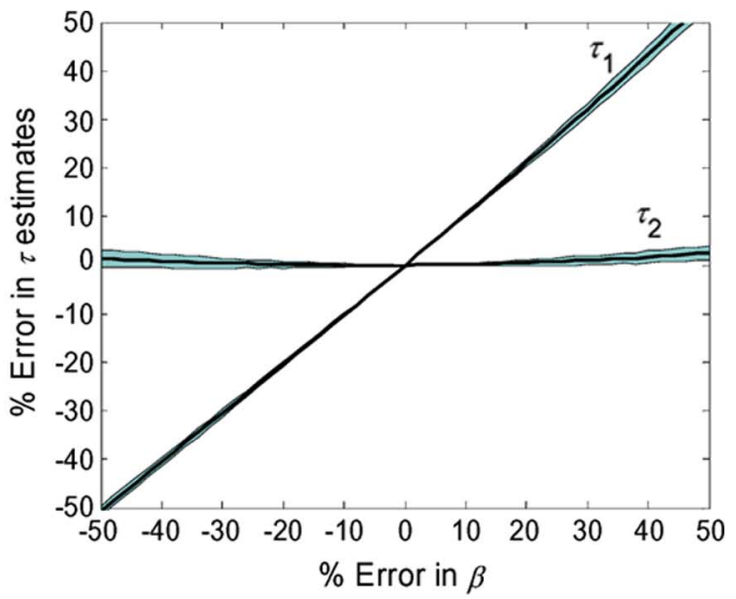

(b)

Fig. 8. Plots of the mean percentage error in parameters $b_{2}, \tau_{1}$, and $\tau_{2}$ due to errors in the assumed value of $\beta$ in the one-parameter $\beta$ algorithm. The shaded regions indicate the $95 \%$ confidence intervals for the estimates. (a) Percentage error in $b_{2}$ versus percentage error in $\beta$. (b) Percentage error in $\tau_{1}$ and $\tau_{2}$ versus percentage error in $\beta$.

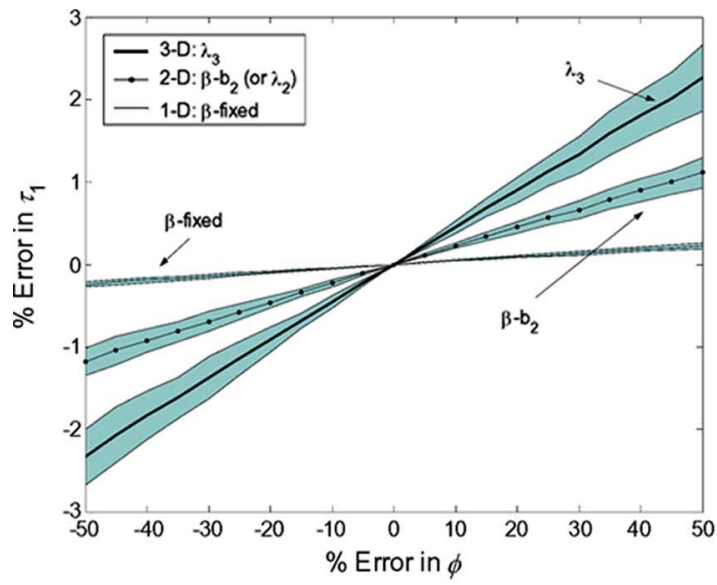

(a)

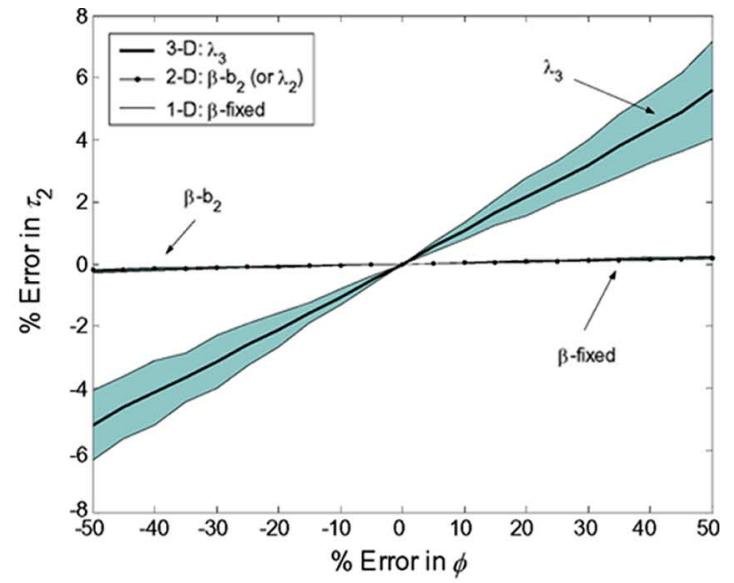

(b)

Fig. 9. Sensitivity of the one-, two-, and three-parameter discrete-time formulation time constant estimates to errors in $\phi$. The shaded regions indicate the $95 \%$ confidence intervals for the estimates. (a) Percentage error in $\tau_{1}$ versus percentage error in $\phi$. (b) Percentage error in $\tau_{2}$ versus percentage error in $\phi$. 


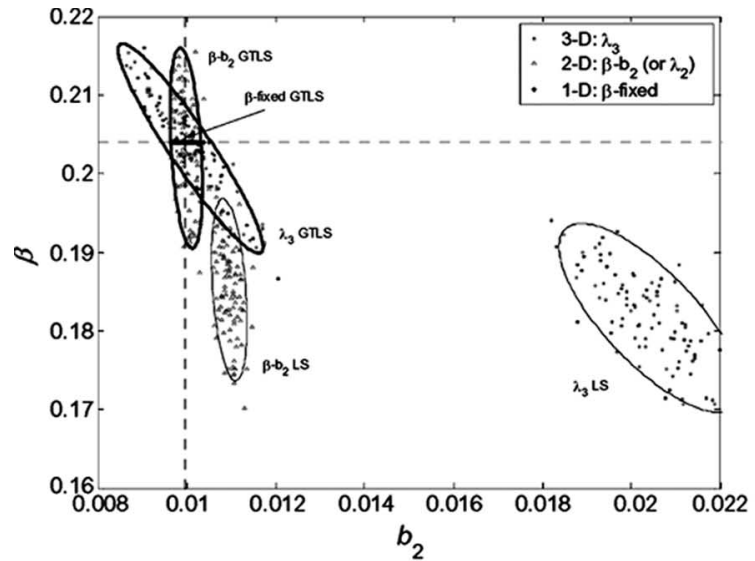

(a)

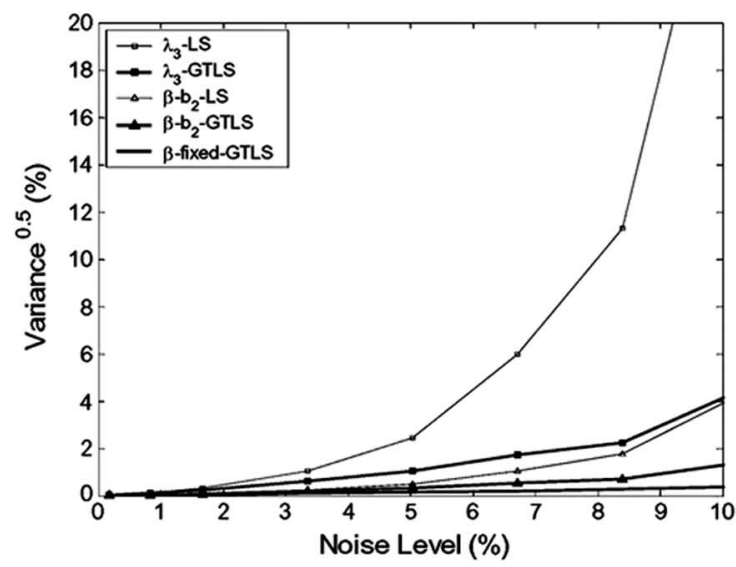

(c)

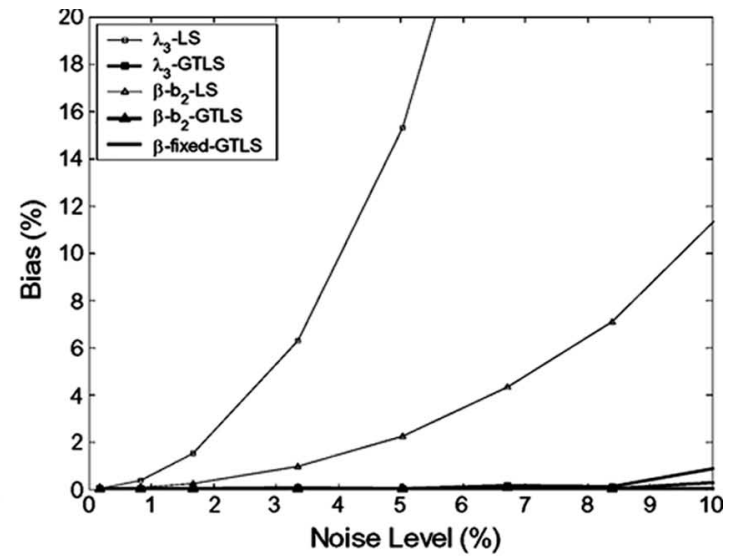

(b)

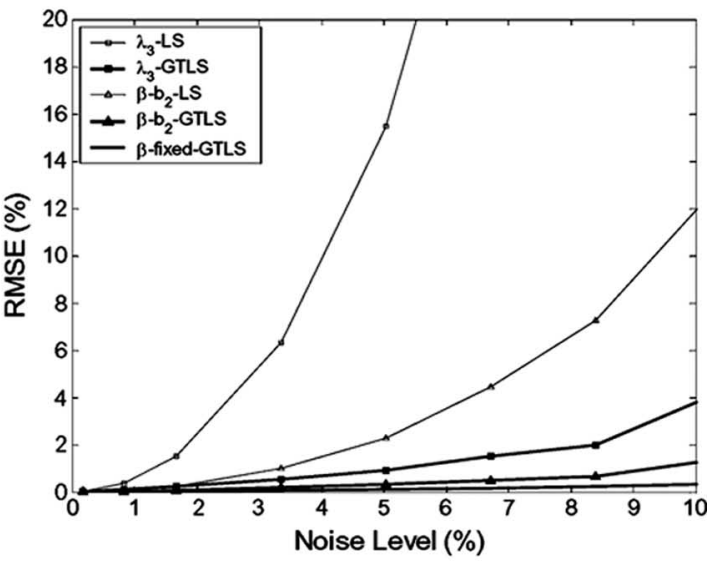

(d)

Fig. 10. Statistical comparison of algorithms in the presence of measurement noise. (a) Covariance ellipses, noise level $=3 \%$. (b) Percentage error due to estimation bias. (c) Percentage error due to estimation variance. (d) Overall percentage root mean square error (RMSE).

2) Sensitivity to Errors in $\phi$ : The other parameter of interest is the noise variance ratio $\phi$. All GTLS algorithms rely on a priori knowledge of this value. The sensitivity of time constant estimates to errors in $\phi$ is illustrated in Fig. 9 under similar experimental conditions to those described in the previous section. It is clear that all formulations are relatively insensitive to errors in $\phi$, with the fixed- $\beta$ implementation being the least sensitive $\left(S_{\tau}^{\phi}<1 / 100\right)$.

\section{Dealing With Offsets on the TTP Measurements}

Analysis of the characterization problem in the presence of offsets on $T_{1}$ and $T_{2}$ reveals that it is the relative offset that is significant. It appears in the regression equations as an additional constant term $c$, i.e.,

$$
\mathbf{X} \theta+c \mathbf{1}_{n}=\boldsymbol{y}
$$

where $\mathbf{1}_{n}$ is an $n \times 1$ vector of $1 \mathrm{~s}$. This relative offset can be identified as part of the LS identification process by augmenting the regression matrix with a vector of $1 \mathrm{~s}$ and rewriting the equation as

$$
\left[\mathbf{1}_{n} \mathbf{X}\right]\left[\begin{array}{l}
c \\
\theta
\end{array}\right]=\boldsymbol{y}
$$

The only difficulty that arises when doing this is that the artificial data $\mathbf{1}_{n}$ introduced into the data matrix is error free with the result that it no longer meets the conditions required for unbiased estimation with GTLS. In these circumstances a variation on GTLS known as RTLS can be employed [19]. This method can handle problems where some of columns of the data matrix are error free and some are not.

Taking the two-parameter $\beta$ formulation (46) as an example and denoting the offset on $T_{1}$ and $T_{2}$ as $\chi_{1}$ and $\chi_{2}$, respectively, the offset-data-based model can be written as

$$
\Delta \bar{T}_{2}^{k}=\beta \Delta \bar{T}_{1}^{k}+b_{2} \Delta \bar{T}_{12}^{k-1}
$$

where

$$
\Delta \bar{T}_{i}^{k} \rightarrow\left(T_{i}^{k}+\chi_{i}\right)-\left(T_{i}^{k-1}+\chi_{i}\right)=\Delta T_{i}^{k}
$$

and

$$
\Delta \bar{T}_{i j}^{k} \rightarrow\left(T_{i}^{k}+\chi_{i}\right)-\left(T_{j}^{k}+\chi_{j}\right)=\Delta T_{i j}^{k}+\left(\chi_{i}-\chi_{j}\right) .
$$

This reduces to the biased model

$$
\Delta T_{2}^{k}=\bar{\beta} \Delta T_{1}^{k}+\overline{b_{2}}\left[\Delta T_{12}^{k-1}+\left(\chi_{1}-\chi_{2}\right)\right]
$$


and, consequently, yields invalid parameter estimates. If, however, a model of the form

$$
\Delta \bar{T}_{2}^{k}=\beta \Delta \bar{T}_{1}^{k}+b_{2} \Delta \bar{T}_{12}^{k-1}+c
$$

is identified, then (65) becomes

$$
\Delta T_{2}^{k}=\beta \Delta T_{1}^{k}+b_{2} \Delta T_{12}^{k-1}+c+b_{2}\left(\chi_{1}-\chi_{2}\right)
$$

allowing the true $\beta$ and $b_{2}$ to be estimated with $c=-b_{2}\left(\chi_{1}-\right.$ $\chi_{2}$ ). Note that the relative offset can also be estimated as

$$
\Delta \chi=\chi_{1}-\chi_{2}=-\frac{c}{b_{2}}
$$

A similar offset extension can be derived for each of the other model formulations considered.

\section{Results}

In this section, the overall noise performance of the different algorithms is investigated using the benchmark problem described in Section I. One hundred run Monte Carlo simulations were performed on this benchmark, with the noise variance ratio $\phi=2$ and used to compute the statistical properties of the parameter estimates for thermocouple 1 noise levels ranging from $0 \%$ to $10 \%$. These were expressed in terms of the corresponding percentage errors in the parameter estimates and are plotted as a function of noise level in Fig. 10. Results are presented for the one-parameter fixed- $\beta$, two-parameter $\beta-b_{2}$, and three-parameter $\lambda$ formulations estimated using GTLS. For comparison purposes, results for conventional LS identification are also included for the two-parameter $\beta-b_{2}$ and the three-parameter formulations. As an example, the distribution of parameter estimates and corresponding covariance ellipses (two standard deviations) are plotted in Fig. 10(a) for the 3\% noise level.

As expected, the LS estimates are strongly biased, whereas the GTLS results are unbiased. Looking at the overall percentage RMSE, it can be seen that the bias error dominates in the LS implementations, whereas the variance error dominates in the GTLS implementations. Since the bias is the main contributor to parameter error, it follows that the GTLS algorithms are superior.

It is also clearly evident in Fig. 10(c) that formulations with fewer degrees of freedom produce parameter estimates with less variance. Consequently, when the time constant ratio is known, the fixed- $\beta$ algorithm is the optimum choice; otherwise, the $\beta-b_{2}$ formulation (or equivalently one of the two-parameter $\lambda$ formulations) is optimum.

\section{CONCLUSION}

This paper has presented a discrete-time formulation of the TTP characterization problem that can be related, through the discrete-parameter ratio $\beta$, to the ratio of thermocouple time constants $\alpha$. This allows a priori knowledge of the time constant ratio to be exploited to obtain characterization algorithms that have low computational complexity and are more robust to measurement noise. Estimation bias is a major source of error in TTP characterization when employing conventional LS, and consequently, methods that can exploit GTLS or equivalent to obtain unbiased estimates are of great value. Unfortunately, the variance of estimates generated by GTLS increases rapidly with noise level limiting direct application to problems with relatively low noise levels. Variance decreases with the number of free parameters, and hence, the availability of the oneparameter fixed- $\beta$ formulation extends the application range when a priori time constant ratio estimates are available.

\section{REFERENCES}

[1] P. Cambray, "Measuring thermocouple time constants: A new method," Combust. Sci. Technol., vol. 45, no. 4, pp. 221-224, 1986.

[2] L. J. Forney and G. C. Fralick, "Two-wire thermocouple: Frequency response in constant flow," Rev. Sci. Instrum., vol. 65, no. 10, pp. 32523257, Oct. 1994.

[3] _ - "Three-wire thermocouple: Frequency response in constant flow," Rev. Sci. Instrum., vol. 66, no. 5, pp. 3331-3336, May 1995.

[4] - "Multiwire thermocouples in reversing flow," Rev. Sci. Instrum., vol. 66, no. 10, pp. 5050-5054, Oct. 1995.

[5] G. H. Golub and C. F. Van Loan, Matrix Computation, 3rd ed. Baltimore, MD: John Hopkins Univ. Press, 1996.

[6] P. C. F. Hung, S. McLoone, G. Irwin, and R. Kee, "A novel approach to two wire thermocouple temperature reconstruction," in Proc. Irish Signals and Syst. Conf., Cork, Ireland, Jun. 2002, pp. 193-198.

[7] — "A total least squares approach to sensor characterisation," in Proc. 13th IFAC Symp. Syst. Identification, Rotterdam, The Netherlands, Aug. 2003, pp. 337-342.

[8] — "Difference equation approach to two-thermocouple sensor characterisation in constant velocity flow environments," Rev. Sci. Instrum. vol. 76, no. 2, p. 024902, Feb. 2005.

[9] — "Unbiased thermocouple sensor characterisation in variable flow environments," in Proc. 16th IFAC World Congr. Autom. Control, Prague, Czech Republic, Jul. 3-8, 2005, CD-ROM.

[10] K. Kar, S. Roberts, R. Stone, M. Oldfield, and B. French, "Instantaneous exhaust temperature measurements using thermocouple compensation techniques," in SAE Transactions, SAE 2004 World Congr. Exhibition, Detroit, MI, Mar. 2004, SAE Paper No. 2004-01-1418.

[11] R. J. Kee, P. G. O'Reilly, R. Fleck, and P. T. McEntee, "Measurement of exhaust gas temperature in a high performance two-stroke engine," $S A E$ Trans. J. Engines, vol. 107, no. 3, pp. 2413-2423, Sep. 1999.

[12] P. G. O'Reilly, R. J. Kee, R. Fleck, and P. T. McEntee, "Two-wire thermocouples: A nonlinear state estimation approach to temperature reconstruction," Rev. Sci. Instrum., vol. 72, no. 8, pp. 3449-3457, Aug. 2001.

[13] H. Pfriem, "Zur Messung veränderlicher Temperaturen von Gasen und Flüssigkeiten," Gen. Ingen., vol. 7, no. 2, pp. 85-92, 1936.

[14] W. C. Strahle and M. Muthukrishman, "Thermocouple time constant measurement by cross power spectrum," J. AIAA Tech. Notes, vol. 14, no. 11, pp. 1642-1644, 1976.

[15] M. Tagawa, K. Kato, and Y. Ohta, "Response compensation of temperature sensors: Frequency-domain estimation of thermal time constants," Rev. Sci. Instrum., vol. 74, no. 6, pp. 3171-3174, Jun. 2003.

[16] M. Tagawa and Y. Ohta, "Two-thermocouple probe for fluctuating temperature measurement in combustion-Rational estimation of mean and fluctuating time constants," Combust. Flame, vol. 109, no. 4, pp. 549560, Jun. 1997.

[17] M. Tagawa, T. Shimoji, and Y. Ohta, "A two-thermocouple probe technique for estimating thermocouple time constants in flows with combustion: In situ parameter identification of a first-order lag system," Rev. Sci. Instrum., vol. 69, no. 9, pp. 3370-3378, Sep. 1998.

[18] S. Van Huffel and J. Vandewalle, "Analysis and properties of the generalised total least squares problem $A X=B$ when some or all columns in A are subject to error," SIAM J. Matrix Anal. Appl., vol. 10, no. 3, pp. 294-315, 1989.

[19] S. Van Huffel and H. Zha, "The restricted total least squares problem: Formulation, algorithm, and properties," SIAM J. Matrix Anal. Appl., vol. 12 , no. 2, pp. 292-309, 1991. 


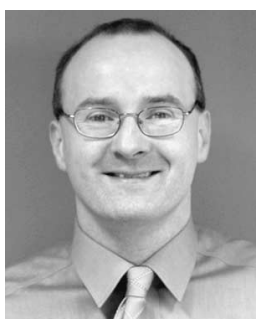

Seán McLoone (S'94-M'96-SM'02) received the M.Eng. degree in electrical and electronic engineering and the Ph.D. degree in control engineering from Queen's University Belfast, Belfast, U.K., in 1992 and 1996, respectively.

$\mathrm{He}$ is a Senior Lecturer with the Department of Electronic Engineering, National University of Ireland Maynooth, Maynooth, Ireland. His research interests are in the general area of data-based modeling and analysis of dynamical systems. This encompasses techniques ranging from classical system identification, fault diagnosis, and statistical process control to modern artificial-intelligence-inspired adaptive learning algorithms and optimization techniques. His research has a strong application focus, with many projects undertaken in collaboration with industry in areas such as process monitoring, control and optimization, time series prediction, and in-line sensor characterization.

Dr. McLoone is a Chartered Engineer, a member of The Institution of Engineers of Ireland, and a member of the Institution of Electrical Engineers. He is currently the Chairman of the U.K. and Republic of Ireland Section of the IEEE, a member of the International Federation of Automatic Control Technical Committee on Cognition and Control, a member of the Machine Learning for Signal Processing Technical Committee of the IEEE Signal Processing Society, and a member of the Royal Irish Academy Engineering Sciences Committee.

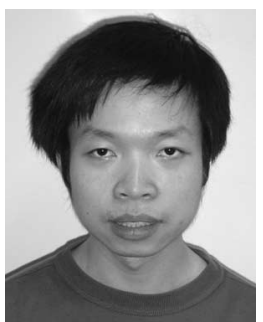

Peter Hung (M'06) was born in Hong Kong. He received the M.Eng. degree in electrical and electronic engineering and the Ph.D. degree in electrical and electronic engineering from Queen's University Belfast, Belfast, U.K., in 2001 and 2005, respectively.

In September 2001, he was awarded a four-year studentship to undertake research at the Virtual Engineering Centre, Queen's University Belfast, on thermocouple sensor fusion and characterization. He is currently a Post-Doctoral Research Fellow with the National University of Ireland Maynooth, Maynooth, Ireland. His research interests include system identification, online and offline signal processing, fault diagnosis, pattern classification, and machine learning. His current projects are in the research fields of fast temperature measurement and integrated circuit testing.

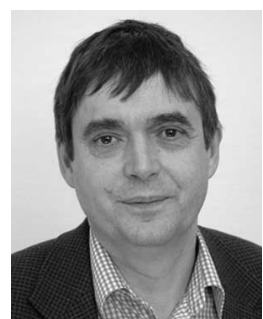

George Irwin (M'83-SM'89-F'04) received the first-class honours degree in electrical and electronic engineering in 1972, the Ph.D. in control theory in 1976, and the D.Sc. degree in 1998 from Queen's University of Belfast, Belfast, U.K.

$\mathrm{He}$ is a Professor of control engineering and the Director of the Virtual Engineering Centre, Queen's University Belfast. His research covers identification, monitoring and control, including neural networks, fuzzy neural systems, and multivariate statistics. He is currently working on wireless networked control systems, fault diagnosis of internal combustion engines, and novel techniques for fast temperature measurement. Much of his work involves industrial collaboration. He was the Technical Director of Anex6 Ltd, which is a spinout company specializing in process monitoring. He has published over 350 research papers and edited six books.

Dr. Irwin is a former Editor-in-Chief of the IFAC Journal Control Engineering Practice and chaired the U.K. Automatic Control Council. He currently chairs the IFAC Publications Committee and serves on the editorial boards of several journals. International recognitions include Honorary Professor at Harbin Institute of Technology, Harbin, China, (1999) and Shandong University, Shandong, China, (2005) and Visiting Professor at Shanghai University, Shanghai, China, (2005-2008). His group has strong collaboration with the Institute of Intelligent Machines, Chinese Academy of Sciences, Heifei, China. He has been elected Fellow of the Royal Academy of Engineering and member of the Royal Irish Academy. He is a Chartered Engineer, a Fellow of the Institution of Electrical Engineers (IEE), and a Fellow of the Institute of Measurement and Control. He has been awarded a number of prizes, including four IEE Premiums, a Best Paper Award from the Czech Academy of Sciences, and the 2002 Honeywell International Medal from the U.K. Institute of Measurement and Control.

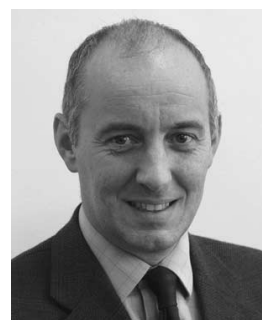

Robert J. Kee received the B.Sc. degree in mechanical engineering in 1981, the Ph.D. degree in 1988 from Queen's University Belfast, Belfast, U.K.

He spent three years in the aircraft industry before returning to Queen's University Belfast to complete his Ph.D. study on stratified scavenged two-stroke engines. He subsequently spent a year in Yamaha, Japan, as a Visiting Researcher and is currently a Senior Lecturer in engineering design in the School of Mechanical and Aerospace Engineering, Queen's University Belfast. He has published/presented 40 papers in journals/international conferences. His initial research concerned the improvement of fuel efficiency and emissions from small engines, which led to a one-year appointment as a Visiting Researcher at Yamaha. Recently, he has focused on the testing and simulation of engines under transient conditions, coupled 1-D and 3-D CFD simulation of unsteady gas flow, fast response temperature measurement, and controlled autoignition in internal combustion engines.

Dr. Kee is a member of the American Society of Automotive Engineers (SAE) Small Engines Technical Committee and the Advanced Powerplant Transactions Selection Committee for the SAE Journal of Engines. He is the recipient of the 2005 SAE Forest R. McFarland Award. 